Article

\title{
Densified Laser-Induced Graphene for Flexible Microsupercapacitors
}

\author{
Jung Bae Lee ${ }^{1}$, Jina Jang ${ }^{1}$, Haoyu Zhou ${ }^{2}$, Yoonjae Lee ${ }^{1}$ and Jung Bin In ${ }^{1,2, *(\mathbb{D})}$ \\ 1 Soft Energy Systems and Laser Applications Laboratory, School of Mechanical Engineering, \\ Chung-Ang University, Seoul 06974, Korea; skzzjd@cau.ac.kr (J.B.L.); jangjina94@cau.ac.kr (J.J.); \\ yolee97@cau.ac.kr (Y.L.) \\ 2 Department of Intelligent Energy and Industry, Chung-Ang University, Seoul 06974, Korea; \\ zhouhaoyu@cau.ac.kr \\ * Correspondence: jbin@cau.ac.kr; Tel.: +82-2-820-5971
}

Received: 20 November 2020; Accepted: 11 December 2020; Published: 13 December 2020

\begin{abstract}
Microsupercapacitors have attracted significant attention due to several of their advantageous characteristics such as lightweight, small volume, and planar structure that is favorable for high mechanical flexibility. Among the various micro supercapacitor forms, those with laser-induced graphene (LIG) electrodes are promising as flexible energy storage devices. While LIG microelectrodes can be fabricated simply by direct laser writing, the capacitance and energy density of these devices are limited because of the relatively low density of LIG, which leads to low surface areas. These limitations could be overcome by densifying the LIG. Here, we report the use of densified laser-induced graphene (d-LIG) to fabricate flexible micro supercapacitors. Interdigitated d-LIG electrodes were prepared by duplicate laser pyrolysis of a polyimide sheet by using a $\mathrm{CO}_{2}$ laser. A PVA- $\mathrm{H}_{2} \mathrm{SO}_{4}$ gel-type electrolyte was then applied to the d-LIG electrode surface to assemble a d-LIG micro supercapacitor. This d-LIG micro supercapacitor exhibited substantially increased capacitance and energy density versus conventional low-density LIG micro supercapacitors. While the d-LIG electrode exhibited a substantial change in resistance when subjected to bending at a radius of $3 \mathrm{~mm}$, the change in the capacitance of the d-LIG micro supercapacitor was negligible at the same bending radius due to reinforcement by the infiltrated poly(vinyl alcohol) (PVA) electrolyte, demonstrating the potential application of d-LIG micro supercapacitors in wearable electronics.
\end{abstract}

Keywords: laser-induced graphene; micro supercapacitor; densification; flexible device

\section{Introduction}

Electrochemical capacitors such as supercapacitors have recently become the focus of intense and broad research efforts to meet the increasing demand for high-performance devices for energy storage [1,2]. Supercapacitors, in particular, have received much attention because of their low costs, long life cycles, rapid charging/discharging capabilities, high power densities, and lightweight. Previous research has focused on developing compact electronics with small sizes, mechanical flexibilities, and high energy and power outputs. Micro supercapacitors (MSCs) are excellent candidates for such micro-scale electronic applications because of several of their characteristic features, including flexibility, portability, and small sizes [3]. Charge storage in MSCs is associated with reversible adsorption/desorption of electrolyte ions at the electrode/electrolyte interface. Devices that operate in this manner are known as electrical double-layer capacitors (EDLCs), which are highly desirable by providing high cycling stability and high-power density but suffer from relatively poor capacitance. Nevertheless, high capacitance in EDLCs can be attained by using electrodes with high conductivities and large surface areas. 
For over a decade, graphene-based materials have demonstrated immense potential for supercapacitors because of their high specific surface areas, micro/meso-porosities, electronic coupling with adsorbates $[4,5]$, and remarkable mechanical and electrical properties. Among this class of materials, three-dimensional (3D) graphene nanomaterials are well suited to serve as electrodes in flexible MSCs [6-8]. 3D graphene nanomaterials are typically obtained by reducing graphene oxide or by chemical vapor deposition, which both generally involve complicated synthetic steps, long processing times, high temperatures, and thus relatively low throughput. Meanwhile, laser-induced graphene (LIG) has become an increasingly useful method to fabricate micro-scale interdigitated electrodes [6,9-11]. One-step direct laser writing of LIG is conducted by irradiating a carbon precursor film (e.g., a polyimide (PI) sheet) with a laser under ambient conditions. The irradiation immediately converts the carbon precursor into a 3D porous graphene nanomaterial (or LIG) by explosive phase change and desorption of byproduct gases. This technique is facile and scalable for fabricating precise micropatterns without a photomask $[10,12,13]$. LIG has been used in various applications including sensors, microfluidic devices, electrocatalytic electrodes, and supercapacitors [14-17]. LIG production can be scaled to enable cost-effective patterning of graphene for miniaturized energy storage devices such as MSCs [9,18-21].

Most efforts to produce LIGs involve the laser pyrolysis of commercial PI sheets, with electrodes produced in this manner being employed in high-performance supercapacitors [22]. Heteroatom doping (with N, S, P, and B atoms) and surface modification with pseudocapacitive materials have been used to enhance the capacitive performances of LIG electrodes $[9,23,24]$. Our group recently developed a novel approach to simultaneously produce densified and nitrogen-doped LIG by duplicate pyrolysis [25]. The as-synthesized densified N-doped LIG was employed as an electrode in supercapacitors. Specifically, the technique involves the initial laser pyrolysis of commercial PI sheets to obtain a LIG. Then, this LIG was coated with an additional layer of PI and subjected to the second laser pyrolysis to generate a densified LIG (d-LIG). Interestingly, this technique not only increased the density of graphene but also elevated the $\mathrm{N}$-content ( 2.65 at.\%) of the graphene. The as-synthesized d-LIG electrode delivered a maximum electrode capacitance of $49.0 \mathrm{mF} / \mathrm{cm}^{2}$ at a current density of $0.2 \mathrm{~mA} / \mathrm{cm}^{2}$ in a three-electrode set up with $1 \mathrm{M} \mathrm{H}_{2} \mathrm{SO}_{4}$ aqueous electrolyte. Interestingly, the capacitance of the d-LIG was estimated to be 6-fold higher than that of LIG prepared by single laser scans (s-LIG). Moreover, a sandwich-type solid-state flexible supercapacitor was assembled by using two parallel d-LIG electrodes separated by a $\mathrm{PVA}^{-} \mathrm{H}_{2} \mathrm{SO}_{4}$ gel electrolyte. This supercapacitor exhibited a device capacitance of $19.8 \mathrm{mF} / \mathrm{cm}^{2}$ at a current density of $0.05 \mathrm{~mA} / \mathrm{cm}^{2}$.

In this study, we have developed a flexible d-LIG MSC by exploiting the high-performance of d-LIG electrodes in an interdigitated format. The duplicate laser pyrolysis technique was used to prepare the d-LIG electrodes; likewise, the MSC electrode pattern area was irradiated twice with a $\mathrm{CO}_{2}$ laser beam. A solid-state flexible MSC was then constructed by applying a gel-type electrolyte $\mathrm{PVA}-\mathrm{H}_{2} \mathrm{SO}_{4}$ to the d-LIG electrodes. The effects of LIG densification on the electrochemical performance of the MSC were investigated, including the retention of d-LIG-MSC capacitance under mechanical strain.

\section{Materials and Methods}

\subsection{Laser Pyrolysis of Polyimide}

Duplicated laser pyrolysis was conducted by using a flying-optic $\mathrm{CO}_{2}$ laser engraver (wavelength: $10.6 \mu \mathrm{m}$, Coryart, Anyang, Korea). The laser beam power was modulated with an optical chopper (MC2000B, Thorlabs.co) with a chopper wheel (MC1F10HP, Thorlabs, Inc., Newton, NJ, USA) operating at a duty cycle of $50 \%$ with an output beam frequency of $1000 \mathrm{~Hz}$. A commercial PI film (thickness: $125 \mu \mathrm{m}$, Kapton ${ }^{\circledR} \mathrm{HN}$, McMaster-Carr, Elmhurst, IL, USA) was used as the initial precursor for the LIG. Figure 1 displays the geometry of the MSC electrode pattern used in this study, and Table 1 shows its dimensions. A raster scanning scheme was used to pyrolyze the pattern area of the PI film, and the gap between the paths of consecutive scanning lines was $125 \mu \mathrm{m}$. 


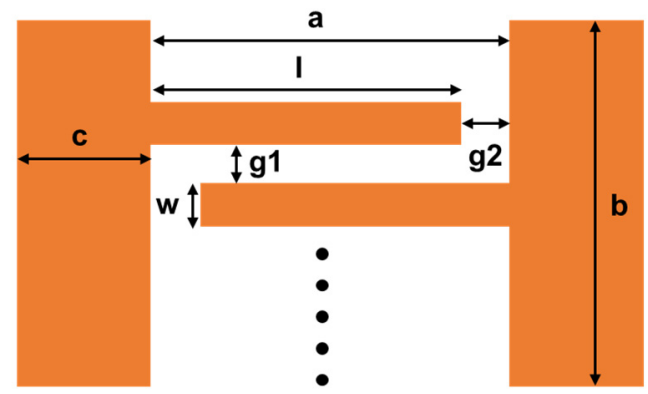

Figure 1. The geometry of the laser-induced graphene (LIG)-micro supercapacitors (MSC) electrode.

Table 1. Dimensions of the LIG-MSC electrode.

\begin{tabular}{cc}
\hline Location & Dimension $(\boldsymbol{\mu m})$ \\
\hline Lateral length of active device area (a) & 5700 \\
Vertical length of active device area (b) & 9060 \\
Pad electrode width (c) & 2660 \\
Finger electrode length (l) & 5350 \\
Finger electrode width (w) & 800 \\
Vertical Gap between finger electrodes (g1) & 250 \\
Gap between finger and pad electrodes (g2) & 350 \\
Number of fingers (N) & 8 \\
\hline
\end{tabular}

Polyamic acid (PAA) was used as the second precursor for the d-LIG. A PAA solution (M: 89,000-98,000, Sigma-Aldrich, St. Louis, MO, USA) was applied to the LIG surface, and the wet LIG was left for $10 \mathrm{~min}$ under ambient conditions to infiltrate the PAA into the LIG. Then, the excess PAA solution was removed by using a spin coater at 1000 RPM for $30 \mathrm{~s}, 1500$ RPM for $20 \mathrm{~s}$, and finally 500 RPM for $20 \mathrm{~s}$. The sample was then annealed in a vacuum oven ( 20 torr) at $100{ }^{\circ} \mathrm{C}$ for $1 \mathrm{~h}$ to completely remove the solvent. Finally, imidization of the PAA was conducted by annealing the dried sample at $250{ }^{\circ} \mathrm{C}$ for $\sim 30 \mathrm{~min}$ by using a hotplate under ambient conditions [25].

s-LIG electrodes were fabricated with the same dimensions as the d-LIG electrodes, but by irradiating the PI sheet with a $\mathrm{CO}_{2}$ laser only once. The optical chopper was not used, and the laser irradiation speed and power were set to $200 \mathrm{~mm} / \mathrm{s}$ and $5.4 \mathrm{~W}$, respectively.

\subsection{Characterizations of the $d-L I G$ Electrodes}

The micromorphology of the d-LIG was investigated by scanning electron microscopy (SEM) (S-3400N, Hitachi High-Tech Co., Tokyo, Japan) and field-emission transmission electron microscopy (FE-TEM) (Tecnai G2 F30 S-TWIN, FEI). TEM samples were prepared by scraping the d-LIG off the electrode substrate and dispersing the resulting d-LIG powder in chloroform. Flakes of the d-LIG were then collected on a TEM grid by dipping the grid in the dispersion. The electrical resistance of the LIG strip was measured by attaching a copper tape to both ends of the strip and applying silver paste (P-100, CANS) to the contact interface.

For bending tests, both ends of the d-LIG strip (LIG area: $0.3 \times 4 \mathrm{~cm}^{2}$ ) were connected to copper tape terminals, and Ga-In eutectic fluid (495425-5G, Sigma-Aldrich) was applied to the LIG-copper interface for minimal change in contact resistance by bending [26]. Bending was achieved by fixing one end of the strip and repeatedly translating the other end to the longitudinal direction by $2 \mathrm{~cm}$ for a specified number of cycles. Electrical resistance was monitored after various cycles of bending, and the resistance retention ratio (\%) was calculated by the equation: $\left(R_{n}-R_{0}\right) / R_{0} \times 100$, where $R_{n}$ is the resistance at the $n$th cycle and $R_{0}$ is the initial resistance of the d-LIG strip before bending. $R_{n}$ and $\mathrm{R}_{0}$ were measured from the d-LIG strip that was in a flat state. Resistance was measured by using a multimeter (34450A, Keysight, Santa Rosa, CA, USA). 


\subsection{Fabrication of Flexible dLIG Microsupercapacitors}

The PVA- $\mathrm{H}_{2} \mathrm{SO}_{4}$ gel electrolyte was prepared by mixing $3 \mathrm{~g}$ of poly(vinyl alcohol) (PVA, M: 89,000-98,000, Sigma-Aldrich) and $3 \mathrm{~mL}$ of $\mathrm{H}_{2} \mathrm{SO}_{4}$ (ACS reagent grade, Sigma-Aldrich) in $30 \mathrm{~mL}$ of deionized water. The electrolyte mixture was then stirred with a magnetic bar at $90{ }^{\circ} \mathrm{C}$ for $6 \mathrm{~h}$, after which the solution became homogeneous and visibly transparent. Then, $60 \mu \mathrm{L}$ of this electrolyte was applied to the surface of the LIG-MSC electrodes. Copper tape was used as the current collector, and silver paste was applied to the contact area to reduce electrical resistance. Kapton tape was used to protect the contact area from the electrolyte. Finally, the sample was dried in a fume hood at $\sim 20^{\circ} \mathrm{C}$ for $24 \mathrm{~h}$ to remove excess water from the electrolyte.

\subsection{Electrochemical Measurements}

The electrochemical performances of the LIG-MSCs were evaluated by using a potentiostat (SP-150, Bio-Logic Science Instruments). Cyclic voltammetry (CV) and galvanostatic charging/discharging (CC) curves were obtained at various scan rates and current densities. Electrochemical impedance spectroscopy (EIS) was conducted by using a sinusoidal potential input with a $10 \mathrm{mV}$ amplitude over a frequency range of $800 \mathrm{kHz}$ to $100 \mathrm{mHz}$. The area-specific capacitance (CA) was calculated from the CC curves based on the following equation [6]: $\mathrm{C}_{\mathrm{A}}=(\mathrm{I} \Delta \mathrm{t}) /(\mathrm{A} \Delta \mathrm{V})$, where $\mathrm{I}$ is the current $(\mathrm{A}), \Delta \mathrm{t}$ is the discharge time $(\mathrm{s}), \Delta \mathrm{V}$ is the potential window $(\mathrm{V})$ for the galvanostatic discharge curve excluding the IR drop, and A is the active area of the LIG (in $\mathrm{cm}^{2}$ ) considering all finger electrodes. The areal energy density $\mathrm{E}_{\mathrm{A}}\left(\mu \mathrm{Wh} \mathrm{cm} \mathrm{cm}^{-2}\right)$ and power density $\mathrm{P}_{\mathrm{A}}\left(\mathrm{mW} \mathrm{cm}^{-2}\right)$ were calculated by using the equations $\mathrm{E}_{\mathrm{A}}=\mathrm{C}_{\mathrm{A}} \times(\Delta \mathrm{V}) /(2 \times 3600)$ and $\mathrm{P}_{\mathrm{A}}=\mathrm{E}_{\mathrm{A}} \times 3600 / \Delta \mathrm{t}$, respectively.

\section{Results and Discussion}

\subsection{Fabrication of d-LIG Microsupercapacitors}

The duplicated laser pyrolysis technique illustrated in Figure 2 was employed to fabricate the d-LIG-MSC electrodes. The first pyrolysis was conducted by irradiating the PI film with a modulated $\mathrm{CO}_{2}$ laser beam with an output power of $\sim 5.8 \mathrm{~W}$ and a scanning speed of $150 \mathrm{~mm} / \mathrm{s}$. The result of this process was a LIG electrode. The optical chopper was used for the first pyrolysis because the use of this particular optical modulation was found to yield reproducible results related to the properties of d-LIG [25]. The LIG was then coated with PAA and subsequently thermally treated to induce the imidization of the PAA, producing a composite layer of LIG and PI. This composite layer was then irradiated with a $\mathrm{CO}_{2}$ laser, which is the second and final pyrolysis step. During this step, the optical chopper was not used. The output laser power was $2.4 \mathrm{~W}$, the laser scanning speed was $200 \mathrm{~mm} / \mathrm{s}$, and the gap for raster scanning was $125 \mu \mathrm{m}$. The laser scanning path was precisely matched to that of the first laser scanning by using aligning marks. As a result, d-LIG-MSC electrodes with the designed geometry (Figure 1) was obtained.

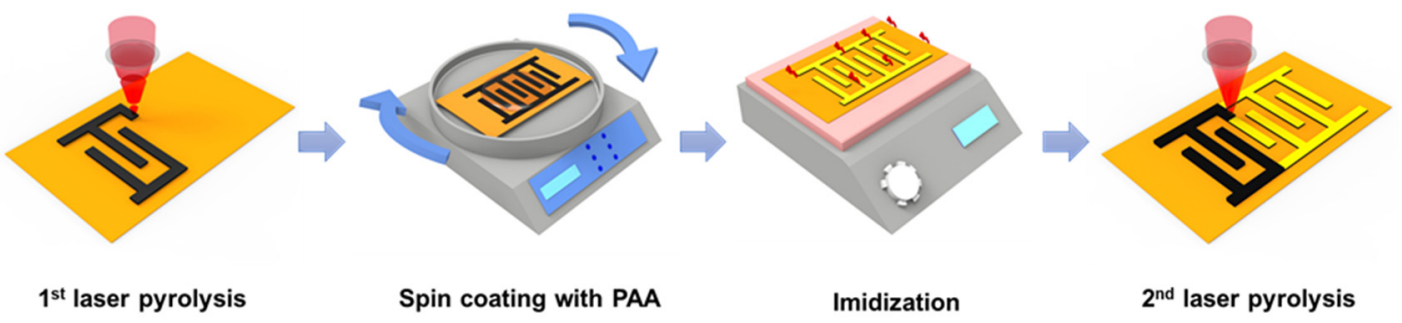

Figure 2. The fabrication process for the d-LIG-MSC electrodes.

The morphology of the LIG-MSC electrode was investigated by optical and electron microscopy. Figure $3 \mathrm{a}, \mathrm{b}$ show optical microscopy images of the LIG produced after the first laser pyrolysis and the d-LIG electrode, respectively. The image of the LIG after the first pyrolysis (Figure 3a) shows traces of 
periodic beam spots that originate from the modulated laser beam $(1 \mathrm{kHz})$. These traces were still visible in the d-LIG (Figure 3b), which was subjected to the second laser pyrolysis. The dimensions of the d-LIG electrode remained similar to those of the LIG. Figure 3c shows an SEM image of the d-LIG electrode, while a magnified SEM image of this material is shown in Figure $3 \mathrm{~d}$. The magnified image reveals that the d-LIG material has a porous microstructure, which is beneficial for supercapacitor electrodes. The SEM image in Figure 3e shows a cross-section of the d-LIG electrode and reveals that a corrugated top structure was generated by raster scanning with the laser. Figure $3 f$ shows a TEM image of a d-LIG flake prepared by scraping the d-LIG electrode. This image shows that the d-LIG consists of graphitic sheets with a myriad of wrinkles.
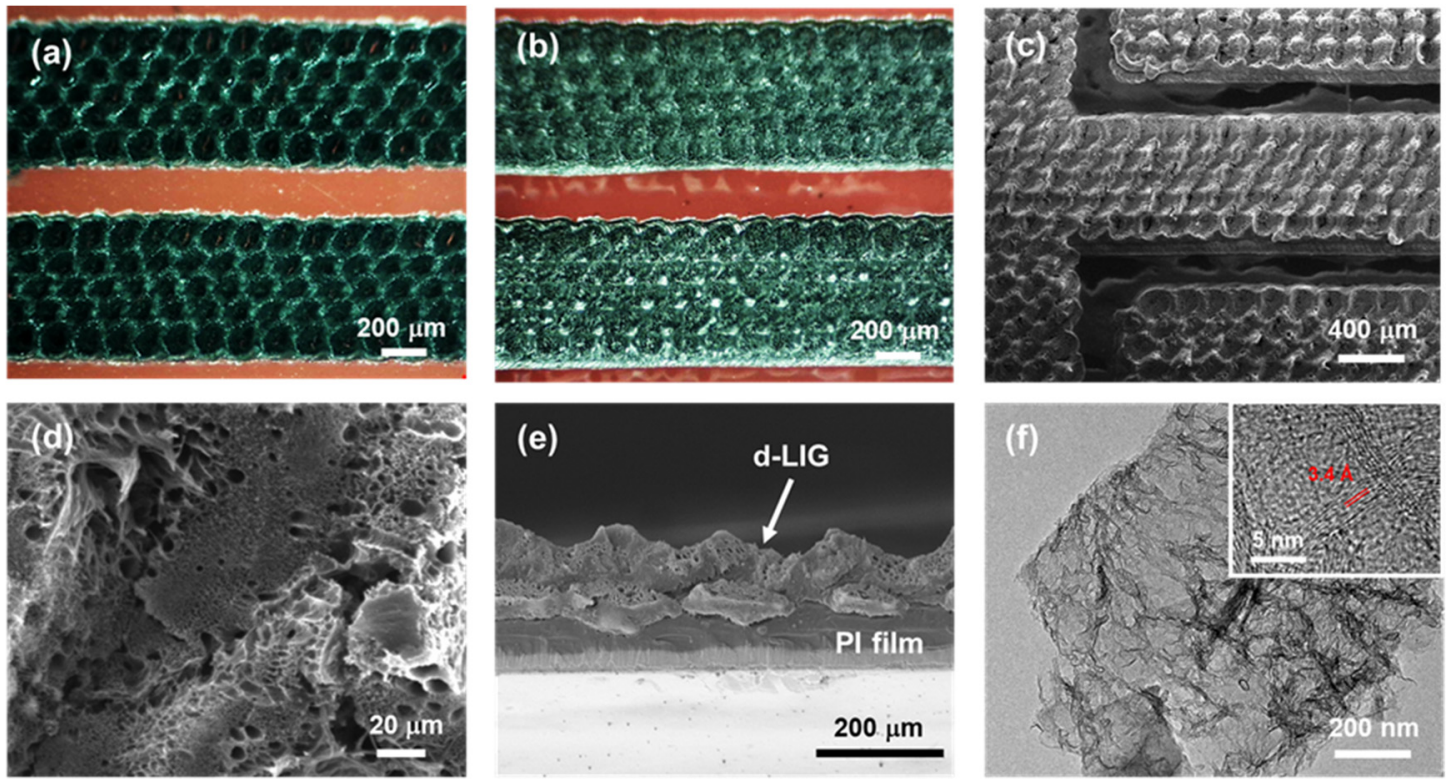

Figure 3. Optical image of an (a) LIG electrode obtained after the first laser pyrolysis and (b) d-LIG-MSC electrode. (c) SEM image of d-LIG-MSC electrodes from a top view, and (d) magnified SEM image of the d-LIG electrode surface. (e) Cross-sectional SEM image of a d-LIG electrode. (f) TEM image of a flake from the d-LIG, with an inset that shows a high-resolution TEM image of the d-LIG flake.

\subsection{Flexibility of $d$-LIG Electrodes}

Mechanical flexibility is an important property of MSC electrodes used in wearable electronics. Ideally, electrodes should retain the electrical and electrochemical properties while the electrode is bent under mechanical strain. We first evaluated the electrical resistance of a d-LIG electrode strip that was repeatedly bent. The same bending test was performed with an s-LIG strip for comparison. Figure 4 a shows the results of the cyclic bending tests and the inset displays a photograph of the loaded and bent LIG strip. The resistances of the d-LIG and s-LIG strips deviated by approximately $10 \%$ and $5 \%$, respectively, over 10,000 cycles. A static bending test with different bending radii was also performed with the s-LIG and d-LIG strips (Figure $4 \mathrm{~b}$ ). In both materials, the resistance tended to increase with decreasing bending radii (see Figure $4 \mathrm{~b}$ inset for definition) due to increased tensile strain in the LIG strip. The d-LIG strip was more sensitive to bending than the s-LIG strip. At the smallest bending radius ( $3 \mathrm{~mm}$ ), the resistance of the d-LIG strip was $\sim 64 \%$ greater than the same material in an unbent state, while the resistance of the s-LIG strip was $\sim 38 \%$ greater than in an unbent state. This can be ascribed to the increased density of the d-LIG, which can suppress the elastic deformation of the porous structure. 
(a)

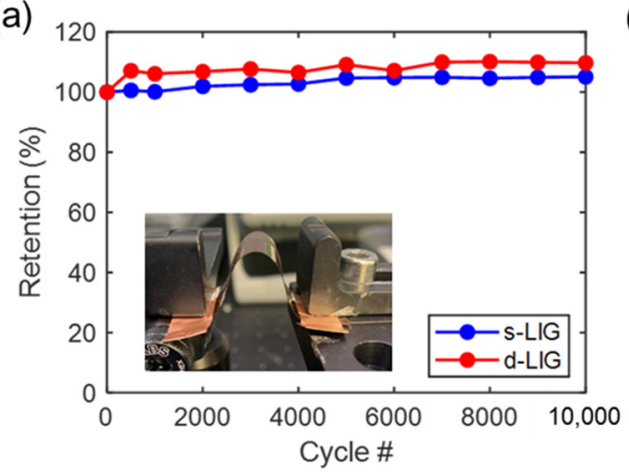

(b)

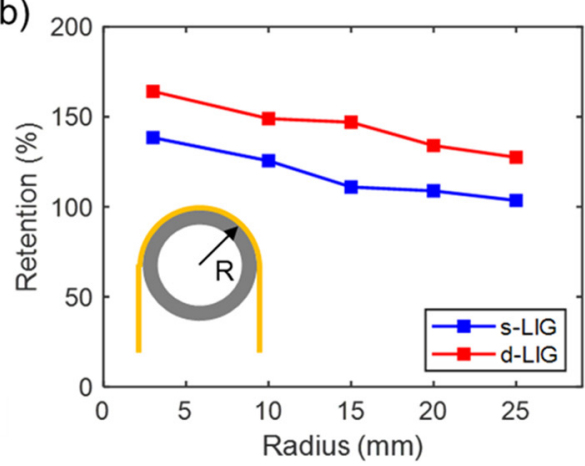

Figure 4. Resistance-based flexibility of bent d-LIG and s-LIG electrode strips. Retention of resistance (\%) (a) after up to 10,000 cyclic bending events, with inset showing a photograph of bending test setup with bent LIG strip, and (b) under bending conditions with different bending radii (a parameter that is defined schematically in the inset).

\subsection{Electrochemical Performance of the d-LIG Microsupercapacitor}

A d-LIG-MSC was fabricated by applying a PVA- $\mathrm{H}_{2} \mathrm{SO}_{4}$ gel-type electrolyte to the surface of the d-LIG-MSC electrode and subsequently evaluated with respect to electrochemical performance, the results of which are displayed in Figure 5. Figure 5a shows CV curves obtained from the d-LIG-MSC at scan rates of $10,20,50,100,200$, and $500 \mathrm{mV} / \mathrm{s}$. The curves exhibit rectangular loops that are typical of graphene-based EDLCs. Figure $5 b$ shows the CC curves measured from the same d-LIG-MSC at current densities of $0.1,0.2,0.5$, and $1 \mathrm{~mA} / \mathrm{cm}^{2}$.

(a)

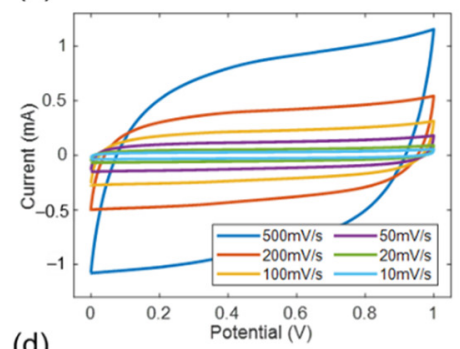

(d)

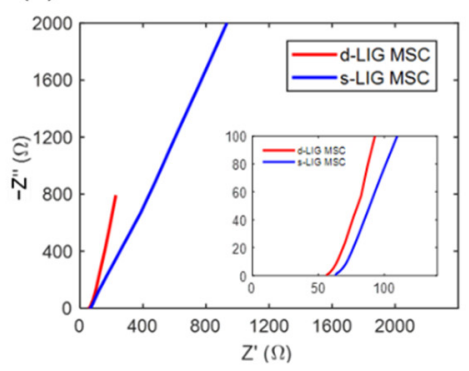

(b)

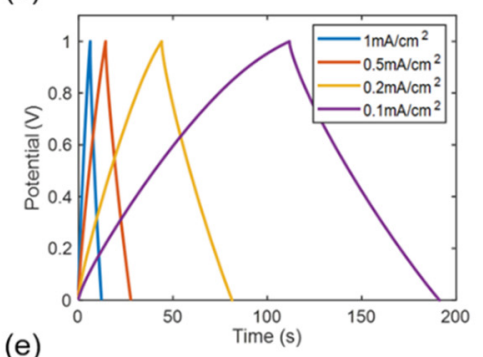

(e)

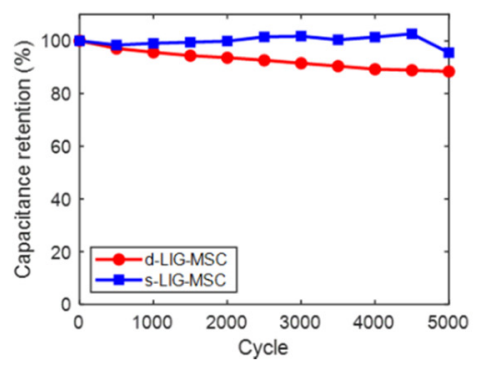

(c)
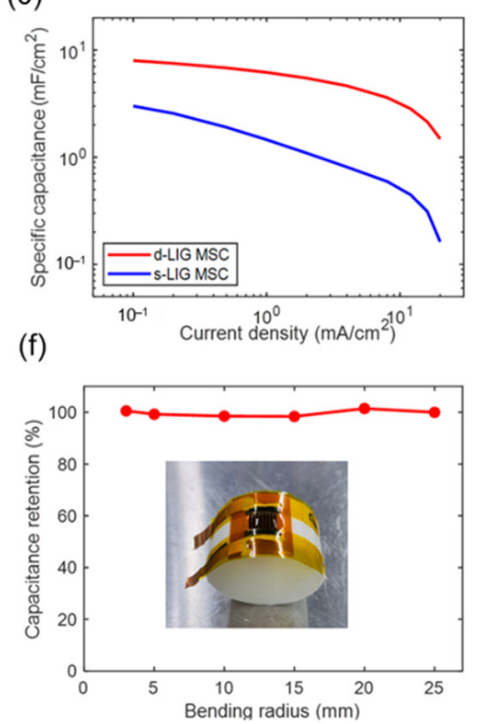

Figure 5. Electrochemical characteristics of the s-LIG-MSC and the d-LIG-MSC. (a) Cyclic voltammetry (CV) and (b) galvanostatic charging/discharging (CC) curves of the d-LIG-MSC at various charging rates. (c) Plot of capacitance versus current density for the s-LIG-MSC and the d-LIG-MSC. (d) EIS curves for the s-LIG-MSC and d-LIG-MSC. Retention of capacitance with (e) cyclic galvanostatic charging/discharging and (f) bending at various radii for the d-LIG-MSC.

The d-LIG-MSC clearly exhibited higher specific capacitance than s-LIG-MSC. The CC testing results in Figure $5 \mathrm{c}$ show a decrease in the area-specific capacitance with increasing current density for both the s-LIG-MSC and d-LIG-MSC, which is attributed to the kinetic limitations of ion transport at high current densities. At the lowest current density of $0.1 \mathrm{~mA} / \mathrm{cm}^{2}$, the specific capacitances of the s-LIG-MSC and d-LIG-MSC were $3.0 \mathrm{mF} / \mathrm{cm}^{2}$ and $8.0 \mathrm{mF} / \mathrm{cm}^{2}$, respectively. Similarly, the 
specific capacitance of the d-LIG-MSC was superior to that of the s-LIG-MSC at higher current densities. For instance, the specific capacitance of the d-LIG was $5.5 \mathrm{mF} / \mathrm{cm}^{2}$ at $2 \mathrm{~mA} / \mathrm{cm}^{2}$, which is $\sim 5$ times as large as that of the s-LIG. Our group previously suggested that the increase in area-specific capacitance with LIG densification originates from laser-induced nitrogen doping and increased loading of LIG ( $\sim 5$ times) in the electrode area of a conventional sandwich-type supercapacitor [25]. This concept can be extended to d-LIG-MSC. Figure $5 \mathrm{~d}$ shows the EIS curves for the s-LIG-MSC and the d-LIG-MSC. Both curves demonstrate capacitive behavior, and, based on the x-intercept of the EIS curve, the equivalent series resistance (ESR) of the d-LIG-MSC was slightly smaller than that of the s-LIG-MSC.

Although the capacitance retention of s-LIG-MSC was superior to that of d-LIG-MSC, the d-LIG-MSC exhibited relatively stable performance after repeated charging/discharging. Figure $5 \mathrm{e}$ shows the retention of capacitance for 5000 cycles of galvanostatic charging/discharging (at a current density of $1 \mathrm{~mA} / \mathrm{cm}^{2}$ ). Rigorous preconditioning of the MSC was not conducted prior to testing, and the capacitance decreased to $88 \%$ during the 5000 cycles. Moreover, the d-LIG-MSC retained performance while bent, maintaining nearly $98 \%$ of capacitance when bent to radii from 3 to $25 \mathrm{~mm}$ (Figure $5 \mathrm{f}$ ). This is in contrast to the relatively large change in the electrical resistance of the d-LIG after bending (Figure $4 \mathrm{~b}$ ). The comparative insensitivity of the electrochemical capacitance to bending possibly arises from the infiltration of the PVA electrolyte that reinforces the LIG structure. This superior performance of the d-LIG-MSC under various bending conditions demonstrates the d-LIG-MSC as a promising energy-storage component in flexible devices.

Figure 6 shows a Ragone plot that compares the performances of the various LIG-based supercapacitors reported in the literature. This plot reveals that densification leads to a significant improvement in the performance of LIG MSC electrodes. The areal energy density of the d-LIG-MSC reported here ranges from 0.05 to $1.1 \mu \mathrm{Wh} \mathrm{cm} \mathrm{cm}^{-2}$ over a corresponding areal power density range of $0.05-10 \mathrm{~mW} \mathrm{~cm}^{-2}$. By comparison, the areal energy density of s-LIG-MSC was at least $62 \%$ lower than those of the d-LIG-MSC over the same power density range. Especially at high power densities, the enhancement of energy density by LIG densification was pronounced. The d-LIG-MSC exhibited superior performance versus other LIG-MSCs that were fabricated by single laser scanning techniques and comparable performance to the B-doped LIG-MSC reported by Peng et al. [9].

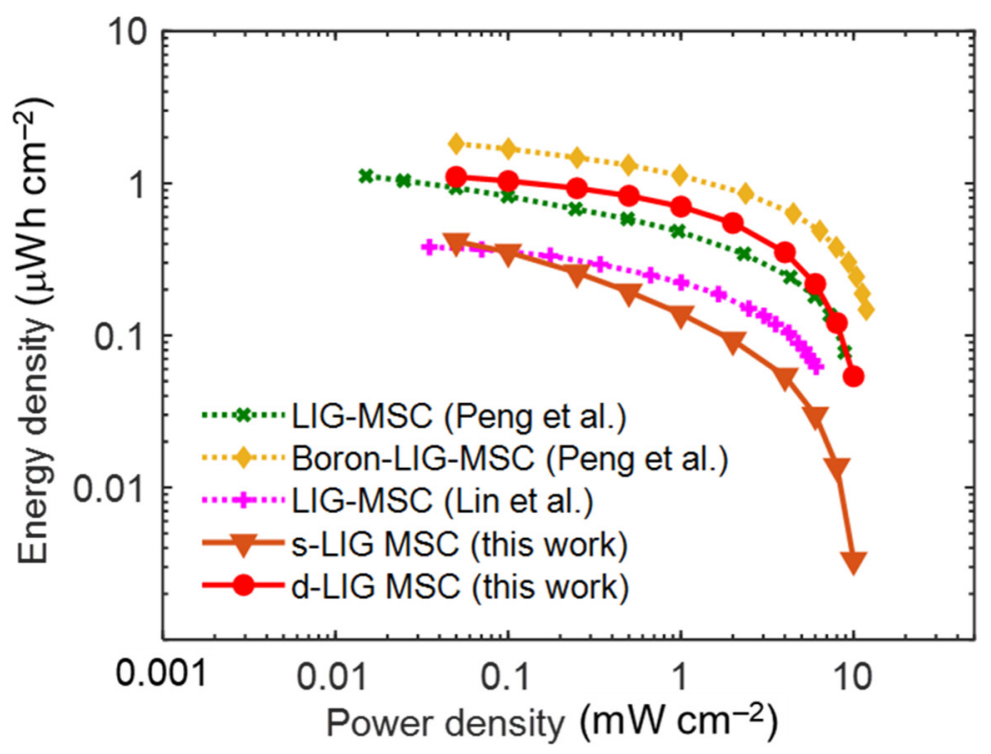

Figure 6. Ragone plot of s-LIG-MSC, d-LIG-MSC, and other LIG-based MSCs fabricated by using a $\mathrm{CO}_{2}$ laser. Origin ${ }^{\circledR}$ software (2019b, OriginLab Co., Northampton, MA, USA) was used to reproduce data reported in Ref. [27] (LIG-MSC, Peng et al.), Ref. [6] (LIG-MSC, Lin et al.), and Ref. [9] (Boron-LIG-MSC, Peng et al.). 


\section{Conclusions}

A high-performance MSC was developed by using interdigitated d-LIG electrodes. d-LIG electrodes were fabricated by laser direct writing an MSC electrode pattern twice with a $\mathrm{CO}_{2}$ laser. This process both increased the density and modified the chemical content of the d-LIG electrode. Moreover, the electrical conductance of d-LIG materials was largely retained after repeated bending. A fully assembled d-LIG-MSC exhibited significantly improved specific capacitance versus a s-LIG-MSC, yielding increased energy density and power density for the d-LIG-MSC. In addition, our investigation suggests d-LIG as a promising graphene-based flexible electrode platform for high-performance hybrid MSC electrodes. The performance of d-LIG-MSCs could be further enhanced by incorporating pseudocapacitive metal oxides and conductive polymers into the d-LIG electrode, as demonstrated in the literature for conventional s-LIG electrodes [16,28,29].

Author Contributions: Conceptualization, J.B.L. and J.B.I.; methodology, J.J. and J.B.I.; validation, J.J.; formal analysis, J.B.L. and J.B.I.; investigation, J.B.L. and H.Z.; writing-original draft preparation, J.B.L., J.J., Y.L., and J.B.I.; writing-review and rditing, Y.L. and J.B.I.; visualization, H.Z.; supervision, J.B.I.; funding acquisition, J.B.I. All authors have read and agreed to the published version of the manuscript.

Funding: This research was supported by the Chung-Ang University Research Scholarship Grants, 2019, and the National Research Foundation of Korea (NRF) grant funded by the Korean government (MSIT) (No. 2009-0082580).

Conflicts of Interest: The authors declare no conflict of interest.

\section{References}

1. Pech, D.; Brunet, M.; Taberna, P.-L.; Simon, P.; Fabre, N.; Mesnilgrente, F.; Conédéra, V.; Durou, H. Elaboration of a microstructured inkjet-printed carbon electrochemical capacitor. J. Power Sources 2010, 195, 1266-1269. [CrossRef]

2. Liu, W.; Song, M.-S.; Kong, B.; Cui, Y. Flexible and Stretchable Energy Storage: Recent Advances and Future Perspectives. Adv. Mater. 2017, 29, 1603436. [CrossRef] [PubMed]

3. Pech, D.; Brunet, M.; Durou, H.; Huang, P.; Mochalin, V.; Gogotsi, Y.; Taberna, P.-L.; Simon, P. Ultrahigh-power micrometre-sized supercapacitors based on onion-like carbon. Nat. Nanotechnol. 2010, 5, 651-654. [CrossRef] [PubMed]

4. Joo, Y.; Kim, M.; Kanimozhi, C.; Huang, P.S.; Wong, B.M.; Roy, S.S.; Arnold, M.S.; Gopalan, P. Effect of Dipolar Molecule Structure on the Mechanism of Graphene-Enhanced Raman Scattering. J. Phys. Chem. C 2016, 120, 13815-13824. [CrossRef]

5. Huang, C.S.; Kim, M.; Wong, B.M.; Safron, N.S.; Arnold, M.S.; Gopalan, P. Raman Enhancement of a Dipolar Molecule on Graphene. J. Phys. Chem. C 2014, 118, 2077-2084. [CrossRef]

6. Lin, J.; Peng, Z.; Liu, Y.; Ruiz-Zepeda, F.; Ye, R.; Samuel, E.L.; Yacaman, M.J.; Yakobson, B.I.; Tour, J.M. Laser-induced porous graphene films from commercial polymers. Nat. Commun. 2014, 5, 5714. [CrossRef] [PubMed]

7. Hsia, B.; Marschewski, J.; Wang, S.; In, J.B.; Carraro, C.; Poulikakos, D.; Grigoropoulos, C.P.; Maboudian, R. Highly flexible, all solid-state micro-supercapacitors from vertically aligned carbon nanotubes. Nanotechnology 2014, 25, 055401. [CrossRef]

8. Dai, L.; Chang, D.W.; Baek, J.-B.; Lu, W. Carbon Nanomaterials for Advanced Energy Conversion and Storage. Small 2012, 8, 1130-1166. [CrossRef]

9. Peng, Z.; Ye, R.; Mann, J.A.; Zakhidov, D.; Li, Y.; Smalley, P.R.; Lin, J.; Tour, J.M. Flexible Boron-Doped Laser-Induced Graphene Microsupercapacitors. ACS Nano 2015, 9, 5868-5875. [CrossRef]

10. In, J.B.; Hsia, B.; Yoo, J.H.; Hyun, S.; Carraro, C.; Maboudian, R.; Grigoropoulos, C.P. Facile fabrication of flexible all solid-state micro-supercapacitor by direct laser writing of porous carbon in polyimide. Carbon 2015, 83, 144-151. [CrossRef]

11. Jang, J.; Yeom, J.W.; Kang, W.K.; Haq, M.R.; Lu, X.; Shin, D.; Kim, S.M.; In, J.B. Laser Pyrolysis of Imprinted Furan Pattern for the Precise Fabrication of Microsupercapacitor Electrodes. Micromachines 2020, 11, 746. [CrossRef] [PubMed] 
12. Cheng, C.; Wang, S.; Wu, J.; Yu, Y.; Li, R.; Eda, S.; Chen, J.; Feng, G.; Lawrie, B.; Hu, A. Bisphenol A Sensors on Polyimide Fabricated by Laser Direct Writing for Onsite River Water Monitoring at Attomolar Concentration. ACS Appl. Mater. Interfaces 2016, 8, 17784-17792. [CrossRef] [PubMed]

13. Cai, J.; Lv, C.; Watanabe, A. Laser Direct Writing and Selective Metallization of Metallic Circuits for Integrated Wireless Devices. ACS Appl. Mater. Interfaces 2018, 10, 915-924. [CrossRef] [PubMed]

14. Stanford, M.G.; Yang, K.; Chyan, Y.; Kittrell, C.; Tour, J.M. Laser-Induced Graphene for Flexible and Embeddable Gas Sensors. ACS Nano 2019, 13, 3474-3482. [CrossRef] [PubMed]

15. Zhang, J.; Zhang, C.; Sha, J.; Fei, H.; Li, Y.; Tour, J.M. Efficient Water-Splitting Electrodes Based on Laser-Induced Graphene. ACS Appl. Mater. Interfaces 2017, 9, 26840-26847. [CrossRef]

16. Li, L.; Zhang, J.; Peng, Z.; Li, Y.; Gao, C.; Ji, Y.; Ye, R.; Kim, N.D.; Zhong, Q.; Yang, Y.; et al. High-Performance Pseudocapacitive Microsupercapacitors from Laser-Induced Graphene. Adv. Mater. 2016, 28, 838-845. [CrossRef]

17. Tao, L.Q.; Tian, H.; Liu, Y.; Ju, Z.Y.; Pang, Y.; Chen, Y.Q.; Wang, D.Y.; Tian, X.G.; Yan, J.C.; Deng, N.Q.; et al. An intelligent artificial throat with sound-sensing ability based on laser induced graphene. Nat. Commun. 2017, 8, 14579. [CrossRef]

18. Cai, J.; Lv, C.; Watanabe, A. Cost-effective fabrication of high-performance flexible all-solid-state carbon micro-supercapacitors by blue-violet laser direct writing and further surface treatment. J. Mater. Chem. A 2016, 4, 1671-1679. [CrossRef]

19. Cai, J.; Lv, C.; Hu, C.; Luo, J.; Liu, S.; Song, J.; Shi, Y.; Chen, C.; Zhang, Z.; Ogawa, S.; et al. Laser direct writing of heteroatom-doped porous carbon for high-performance micro-supercapacitors. Energy Storage Mater. 2020, 25, 404-415. [CrossRef]

20. Cai, J.; Lv, C.; Watanabe, A. Laser direct writing of high-performance flexible all-solid-state carbon micro-supercapacitors for an on-chip self-powered photodetection system. Nano Energy 2016, 30, 790-800. [CrossRef]

21. Shen, D.; Zou, G.; Liu, L.; Zhao, W.; Wu, A.; Duley, W.W.; Zhou, Y.N. Scalable High-Performance Ultraminiature Graphene Micro-Supercapacitors by a Hybrid Technique Combining Direct Writing and Controllable Microdroplet Transfer. ACS Appl. Mater. Interfaces 2018, 10, 5404-5412. [CrossRef] [PubMed]

22. Ye, R.; James, D.K.; Tour, J.M. Laser-Induced Graphene. Acc. Chem. Res. 2018, 51, $1609-1620$. [CrossRef] [PubMed]

23. Yang, W.; Liu, Y.; Li, Q.; Wei, J.; Li, X.; Zhang, Y.; Liu, J. In situ formation of phosphorus-doped porous graphene via laser induction. Rsc. Adv. 2020, 10, 23953-23958. [CrossRef]

24. Singh, S.P.; Li, Y.; Zhang, J.; Tour, J.M.; Arnusch, C.J. Sulfur-Doped Laser-Induced Porous Graphene Derived from Polysulfone-Class Polymers and Membranes. ACS Nano 2018, 12, 289-297. [CrossRef]

25. Kim, K.Y.; Choi, H.; Van, T.C.; In, J.B. Simultaneous densification and nitrogen doping of laser-induced graphene by duplicated pyrolysis for supercapacitor applications. J. Power Sources 2019, 441, 227199. [CrossRef]

26. Choi, H.; Nguyen, P.T.; In, J.B. Laser transmission welding and surface modification of graphene film for flexible supercapacitor applications. Appl. Surf. Sci. 2019, 483, 481-488. [CrossRef]

27. Peng, Z.; Lin, J.; Ye, R.; Samuel, E.L.; Tour, J.M. Flexible and stackable laser-induced graphene supercapacitors. ACS Appl. Mater. Interfaces 2015, 7, 3414-3419. [CrossRef]

28. Ye, R.Q.; Peng, Z.W.; Wang, T.; Xu, Y.N.; Zhang, J.B.; Li, Y.L.; Nilewski, L.G.; Lin, J.; Tour, J.M. In Situ Formation of Metal Oxide Nanocrystals Embedded in Laser-Induced Graphene. ACS Nano 2015, 9, 9244-9251. [CrossRef]

29. Choi, H.; Nguyen, P.T.; Tran, C.V.; In, J.B. Micro-patterned metal current collectors for high aspect ratio flexible graphene supercapacitors. Appl. Surf. Sci. 2020, 510, 145432. [CrossRef]

Publisher's Note: MDPI stays neutral with regard to jurisdictional claims in published maps and institutional affiliations.

(C) 2020 by the authors. Licensee MDPI, Basel, Switzerland. This article is an open access article distributed under the terms and conditions of the Creative Commons Attribution (CC BY) license (http://creativecommons.org/licenses/by/4.0/). 